\title{
Loose Bernoullicity is preserved under exponentiation by integrable functions
}

\author{
MAURICE H. RAHE AND DANIEL J. RUDOLPH \\ Department of Mathematics, Texas A\&M University, College Station, TX 77843, \\ USA; Department of Mathematics, University of Maryland, College Park, \\ Maryland 20742, USA
}

(Received 23 November 1985)

Abstract. It is known that if $\Omega$ is a Lebesgue space, $T: \Omega \rightarrow \Omega$ is a loosely Bernoulli transformation, and $L$ is a fixed non-zero integer, then the transformation $S=T^{L}$ will again be loosely Bernoulli on each ergodic component. In this note, the above stated result is extended to include the case where $L$ is an arbitrary integrable integer-valued function on $\Omega$.

Let $(\Omega, \mathscr{F}, \mu)$ be a Lebesgue space and $T: \Omega \rightarrow \Omega$ an invertible, ergodic, measurepreserving transformation. For $L$ an integrable integer-valued function on $\Omega$, we consider the transformation $U(\omega)=T^{L(\omega)}(\omega)$. In general, $U$ will not be invertible and will not preserve $\mu$. Moreover, not every point $\omega \in \Omega$ will belong to a bilateral $U$-orbit, i.e. a set $S=\left\{\omega_{i}: i \in \mathbb{Z}\right\}$ where $U\left(\omega_{i}\right)=\omega_{i+1}$.

On the other hand, it was shown in [1] that with the above hypothesis there exists a set $\Omega_{1}$ of full measure (of points satisfying a certain finiteness condition) and the set $A \subseteq \Omega_{1}$ of points which also belong to a bilateral $U$-orbit has strictly positive measure. Moreover, the transformation $U=T^{L}$ restricted to $A$ is invertible and preserves $\mu_{A}$, but may not be ergodic. In this article we note that the behavior of $U$ on almost all ergodic components can be explicitly described.

More precisely, we claim that there exists a $U$-invariant set $B \subseteq A$ with $\mu_{A}(B)=1$ such that there are finitely many (possibly zero) sets $C$ with $\mu(C)>0$ and $U$ restricted to $C$ aperiodic ergodic; and all other ergodic components of $U$ in $B$ are finite rotations. To see this, note that if there exists $C \subseteq A$ with $\mu(C)>0$ and $U(C)=C$, then by ergodicity $\mu$-a.e. $T$-orbit contains points in $C$ and hence a complete $U$-orbit lying in $C$. However, by [1, Theorem 2(b)] there can be at most finitely many aperiodic $U$-orbits (cardinality of orbit not finite) on a $T$-orbit. The number $m$ of aperiodic $U$-orbits on a $T$-orbit is an upper bound for the number of such sets $C$. Thus the set $B$ consists of a union of all periodic $U$-orbits and a finite number of such $C$, where $U$ restricted to $C$ is aperiodic.

We now describe the behavior of $U$ on the aperiodic components of $B$. For any $U$-invariant $C \subseteq B$ with $\mu(C)>0$ for which $U$ restricted to $C$ is aperiodic ergodic, let $L^{\prime}: C \rightarrow \mathbb{Z}$ be the integer-valued function such that $\left.U\right|_{C}=\left(T_{C}\right)^{L^{\prime}}$, where $\left.U\right|_{C}$ is the restriction and $T_{C}$ is the induced transformation. Clearly $\left|L^{\prime}\right| \leq|L|$. Moreover, it follows from aperiodicity that the values $\sum_{i=1}^{n} L^{\prime}\left(U^{i} \omega\right), n=1,2, \ldots$, are disjoint 
for $\mu_{C^{-a}}$.e. $\omega$, hence

$$
\limsup _{n \rightarrow \infty} \frac{1}{n}\left|\sum_{i=1}^{n} L^{\prime}\left(U^{i} \omega\right)\right| \geq \frac{1}{2}
$$

By the ergodic theorem, we must therefore have $\left|\int L^{\prime} d \mu_{C}\right| \neq 0$. Assume $\int L^{\prime} d \mu_{C}>0$, the other case being similar.

By ergodicity we can choose a set $D$ with $\mu_{C}(D)>\frac{1}{2}$ and a positive integer $N$ such that for $n \geq N$ we have

$$
\frac{1}{n} \sum_{i=1}^{n} L^{\prime}\left(U^{i} \omega\right)>\frac{1}{2} \int L^{\prime} d \mu_{C}>0 \quad \text { for } \omega \in D .
$$

Now choose $E \subseteq D, \mu_{C}(E)>0$, so that for $\omega \in E$ we have $\inf \left\{i>0: U^{i} \omega \in E\right\} \geq N$. Then the function $\bar{L}: E \rightarrow \mathbb{Z}$ such that $\left(\left.U\right|_{C}\right)_{E}=U_{E}=\left(T_{E}\right)^{\bar{L}}$ satisfies $\bar{L}>0$ and $\int \bar{L} d \mu_{E}=\int L^{\prime} d \mu_{C}$.

We now observe that $T_{E}$ is a factor of a tower transformation over $U_{E}$. Let $\hat{E}$ be the subset of $E \times\{0,1,2, \ldots\}$ below the graph of $\bar{L}$. Let $\hat{\mu}$ be defined as

$$
\hat{\mu}(F)=\sum_{i=0}^{\infty} \mu_{E}\left(F_{j}\right)\left(\int \bar{L} d \mu_{E}\right)^{-1}
$$

where $F_{j}$ denotes the section of $F$ at $j$. Let $\hat{U}: \hat{E} \rightarrow \hat{E}$ be defined by $\hat{U}(\omega, i)=(\omega, i+1)$ if $0 \leq i<\bar{L}(\omega)-1$, while $\hat{U}(\omega, \bar{L}(\omega)-1)=\left(U_{E}(\omega), 0\right)$. Then it is well known that $\hat{U}$ is an ergodic transformation on $\hat{E}$ which preserves $\hat{\mu}$, and

$$
\hat{\mu}(E \times\{0\})=\left(\int \bar{L} d \mu_{E}\right)^{-1}=\left(\int L^{\prime} d \mu_{C}\right)^{-1} .
$$

Define $\Phi: \hat{E} \rightarrow E$ to be the map taking $(\omega, i)$ to $\left(T_{E}\right)^{i} \omega$. Note that $T_{E} \circ \Phi=\Phi \circ \hat{U}$, so $\hat{U}$ is a skew product over $T_{E}$. As mentioned earlier, the number $m$ of aperiodic $U_{E}$-suborbits on a $T_{E}$-orbit is finite and, by ergodicity, constant almost everywhere. Since $\bar{L}>0$ and $U_{E}$ is ergodic, it is easy to see that $m=\int \bar{L} d \mu_{E}$. (Hence $\int L^{\prime} d \mu_{C}$ must be a positive integer. See also [2; Proposition 10].) Moreover $\hat{U}$ is an $m$-point extension of $T_{E}$, i.e. $\hat{U}$ is a skew product of $T_{E}$ with the symmetric group on the integers $\{1,2, \ldots, m\}$. Since one can write each $m$-point extension of $T_{E}$ as the transformation induced on the set $E \times\{1,2, \ldots, m\}$ by an $m$-point extension of $T$ (where the skewing on $\Omega-E$ is the identity), we see that $\left.U\right|_{C}$ is Kakutani equivalent to a finite extension of $T$. In particular, let $(\hat{T}, \hat{\Omega})$ denote the $m$-point extension of $T$. We have that $\left.U\right|_{C}$ induces $U_{E}$, where $E$ has relative measure $\mu(E) / \mu(C)$ in $C$. Moreover, $\hat{T}$ induces $\hat{U}$, which in turn induces $U_{E}$, where $E$ has relative measure $\mu(E) / m$ in $\hat{\Omega}$. Then if $m>1$ or $\mu(E)<1$, we have that $\hat{T}$ induces $\left.U\right|_{C}$ from [3, lemma 1.3]. If $m=1$ and $\mu(E)=1$, then $\left.U\right|_{C} \simeq T$, so trivially $\left.U\right|_{C}$ is induced by an $m$-point extension of $T$. We summarize these results in the following theorem.

THEOREM. Let $(\Omega, \mathscr{F}, \mu)$ be a Lebesgue space and $T: \Omega \rightarrow \Omega$ an invertible ergodic measure-preserving transformation. Let $L$ be an integrable integer-valued function. Then for $U(\omega)=T^{L(\omega)}(\omega)$, there is a maximal $U$-invariant set $A \subseteq \Omega$, with $\mu(A)>0$, on which $U$ is invertible and preserves $\mu_{A}$. Moreover, there is a set $B \subseteq A$ with $\mu_{A}(B)=1$ such that there are at most finitely many sets $C \subseteq B$ with $\mu(C)>0$ and $\left.U\right|_{C}$ aperiodic 
ergodic. On each of these, $\left.U\right|_{C}$ is induced by a finite extension of $T$. For all other $C \subseteq B$ with $\left.U\right|_{C}$ ergodic, $\left.U\right|_{C}$ is a finite rotation.

COROLlaRY. If $T$ is loosely Bernoulli and $L$ is an arbitrary integrable function, $T^{L}$ is loosely Bernoulli on each ergodic component.

Proof. By [3, Lemma 6.6], loose Bernoullicity is preserved under inducing. By [4] and [3; Corollary 7.9], it is also preserved under finite extensions.

\section{REFERENCES}

[1] J. C. Kieffer \& M. H. Rahe. The pointwise ergodic theorem for transformations whose orbits contain or are contained in the orbits of a measure-preserving transformation. Canad. J. Math. XXXIV, (No. 6) (1982), pp. 1303-1318.

[2] J. Neveu. Temps d'arrêt d'un système dynamique. Z. Wahrscheinlichkeitstheorie verw. Geb. 13 (1969), pp. 81-94.

[3] D. S. Ornstein, D. J. Rudolph \& B. Weiss. Equivalence of Measure Preserving Transformations. Mem. Amer. Math. Soc. 262, 37 (1982).

[4] D. J. Rudolph. If a finite extension of a Bernoulli shift has no finite rotation factors, it is Bernoulli. Israel J. Math. 30 (1978), pp. 193-206. 